\title{
Adhesivos de cianoacrilato en cirugía oral y maxilofacial
}

\section{Cyanoacrylate adhesives in oral and maxillofacial surgery}

\author{
Alex Vargas $^{1}$, Camila Foncea ${ }^{1,2^{*}}$, Andrés Campolo ${ }^{1,2}$ \\ 1. Departamento Cirugía Oncológica y Maxilofacial. \\ Facultad de Medicina, Pontificia Universidad \\ Católica de Chile. Santiago, Chile. \\ 2. Residente Cirugía Maxilofacial, Pontificia \\ Universidad Católica de Chile. Santiago, Chile. \\ ${ }^{*}$ Correspondencia a: Dra. Camila Foncea \\ (cfoncea1@uc.cl) | Marcoleta \#352 patio interior. \\ Santiago, Chile. I (56-2) 2354-3720 \\ Trabajo recibido el 05/07/2016. Aprobado para su \\ publicación el 21/09/2016

\begin{abstract}
RESUMEN
Los adhesivos de cianoacrilatos (ACA) son materiales sintéticos con propiedades adhesivas. Al ser aplicados en los tejidos polimerizan uniéndose con el tejido subyacente. Desde la década de los 70' se han explorado sus aplicaciones quirúrgicas para el cierre de heridas y fístulas, control de sangrado y fijación de injertos, entre otros, siendo su uso como alternativa para el cierre de heridas en piel y mucosas uno de los más estudiados. Los ACA presentan un limitado grado de absorción, sin evidencia de efectos tóxicos sistémicos. Tienen la ventaja de ser aplicados de forma rápida, indolora, con efecto antibacteriano y hemostático según los reportes de la literatura, pero presentan una reducida fuerza de tensión. El objetivo de esta revisión de la literatura es describir los usos y aplicaciones de los ACA, con enfoque en la cirugía oral y maxilofacial, evaluando de forma crítica sus aplicaciones.
\end{abstract} \\ PALABRAS CLAVE \\ Cianoacrilatos, suturas, cierre de heridas, cirugía oral.
}

Rev. Clin. Periodoncia Implantol. Rehabil. Oral Vol. 10(2); 107-110, 2017.

\section{ABSTRACT}

The cyanoacrylate adhesives (ACA) are synthetic materials with adhesive properties. When is applied in tissues, it polymerizes and bonds with the underlying tissue. Since the 70s' have been explored their surgical applications for closing wounds, fistulas, bleeding control, and graft fixation, among others. Its use as an alternative for closing wounds in skin and mucous is one of the most studied. The ACA have a limited absorption degree, with no evidence of systemic toxic effects. They have the advantage of being applied quickly, painlessly, with antibacterial effect and hemostatic according to the report of literature, but with reduced tensile strength. The objective of this literature review is to describe the use and applications of ACA, with focus on oral and maxillofacial surgery, with a critically evaluation of their applications.

KEY WORDS

Cyanoacrylates, sutures, wound closure, oral surgery.

Rev. Clin. Periodoncia Implantol. Rehabil. Oral Vol. 10(2); 107-110, 2017.

\section{INTRODUCCIÓN}

Los adhesivos de cianoacrilatos (ACA) son materiales sintéticos que polimerizan en contacto con agua o humedad. Fueron descritos por primera vez en 1949 por $\operatorname{Ardis}^{(1)}$, pero fue Coover quien en $1959^{(2)}$ señaló sus propiedades adhesivas y sugirió su posible aplicación quirúrgica. Entre algunas de las aplicaciones de los ACA destaca su uso en el cierre de heridas y fístulas, control de sangrado gastrointestinal y fijación de injertos óseos y cutáneos; estando contraindicado en heridas infectadas, pacientes inmunocomprometidos y en alergia conocida a cianoacrilatos y formaldehido ${ }^{(3)}$.

Su uso como alternativa para el cierre de heridas en piel y mucosas ha sido uno de los más estudiados. Dentro de sus principales ventajas destacan su rápida aplicación, resistencia a la infección, propiedades hemostática y el no requerir su retiro posterior ${ }^{(3)}$, a diferencia de las suturas convencionales no reabsorbibles.

En el área de odontología y cirugía maxilofacial, ha sido utilizado para el manejo de hipersensibilidad dentinaria ${ }^{(4)}$, en combinación con doximicina para el tratamiento sintomático de úlceras aftosas recurrente ${ }^{(5)}$, embolización de la arteria mandibular y como medida local del manejo del sangrado post operatorio secundario a exodoncia compleja de terceros molares $^{(6)}$, entre otros.

Existe cierta tendencia por parte de los clínicos a preferir el uso de ACA en determinadas situaciones clínicas ${ }^{(7)}$. Por esta razón, el objetivo de esta revisión de la literatura es describir los usos y aplicaciones de los ACA, con enfoque en la cirugía oral y maxilofacial, evaluando de forma crítica su utilidad y aplicaciones en esta área.

\section{Composición y propiedades de los cianoacrilatos}

Químicamente los ACA pertenecen a la familia de los alquil cianoacrilatos $\left(\mathrm{CH}_{2}=\mathrm{C}(\mathrm{CN}) \mathrm{COOR}\right)$. Se sintetizan por la reacción de formaldehido con alquil-cianoacrilato, obteniendo un pre-polímero que a altas temperaturas es depolimerizado a un monómero de presentación líquida ${ }^{(8)}$.

Al ser aplicados en los tejidos y aún en presencia de humedad, polimerizan en segundos por una reacción exotérmica al contacto con
aniones, especialmente iones hidroxilo o grupos amino de proteínas ${ }^{(9-11)}$, formando una unión adhesiva con el tejido subyacente mediante una película delgada y sólida ${ }^{(12)}$.

Los diferentes tipos de ACA se caracterizan según el largo de la cadena lateral de alquilo (-COOR), clasificándose en adhesivos de cadena corta, metil-CA (una cadena) y etil-CA (dos), y de cadena larga, butil-CA (cuatro), isoamil-CA (cinco) y octil-CA (ocho) ${ }^{(3,13)}$. Metil-CA, es el adhesivo original de menor tamaño, que polimeriza en un material duro. Los análogos de cadena lateral más larga como octil y butil son más resilientes, con mayor resistencia a la tensión ${ }^{(3,11,14)}$. Se describe que octil-CA es el más fuerte y flexible de todos, con una resistencia tridimensional 4 veces mayor a la del butil-CA ${ }^{(15)}$

Los ACA son biodegradables, y su remoción del sitio de aplicación depende del ataque hidrolítico de los enlaces de carbono, obteniendo formaldehido y cianoacrilato(3). Su absorción es mínima y se descaman de la superficie de piel y mucosa después de 5 a 10 días de su aplicación ${ }^{(14,16)}$.

\section{Toxicidad sistémica}

Los primeros ACA de cadena corta fueron discontinuados por asociarse a una reacción tóxica en los tejidos. En su lugar, apareció el 
$\mathrm{N}$-butil-2-cyanoacrylato (Histoacry|®), demostrando menor toxicidad y rápida adhesión a tejidos blandos y duros ${ }^{(17)}$, siendo aprobado para su uso clínico a inicios de $1996^{(18)}$. Más tarde, en 1998, la FDA aprobó el uso de octil-2-ciniacrilato (Dermabond, Ethicon $®$ ) para la aproximación de heridas quirúrgicas y traumáticas con poca tensión ${ }^{(7,19)}$.

El efecto tóxico de los ACA en los tejidos depende de sus productos de degradación y la velocidad con la cual estos se producen ${ }^{(19,20)}$. La concentración de estos productos es proporcional al rango de degradación del compuesto original. Los ACA de cadena larga son menos reactivos que los de cadena corta y su degradación mas lenta resulta en menos concentración de sus productos de degradación en los tejidos locales, generando menos inflamación ${ }^{(3,8,13,16,21)}$, siendo los ACA de butil y octil-CA las alternativas menos toxicas ${ }^{(9)}$.

Se ha descrito un limitado grado de absorción dérmica luego de su aplicación tópica(22). En modelos animales, los ACA no han demostrado tener efecto tóxicos sistémicos cuando son aplicados de forma tópica o subcutánea ${ }^{(23)}$, sin evidencia de producir alteraciones a nivel renal o hepático ${ }^{(24)}$

Específicamente, Inal et al, el 2006 evaluaron en un modelo animal el efecto sistémico del N-Butil-2-CA aplicado en mucosa oral de ratas, sin encontrar diferencias estadísticamente significativas en los niveles de nitrógeno ureico y creatinina entre el control y los 2, 14, 21, y 65 días post operatorio(18). Tampoco se han reportado efectos carcinogénicos para butil e isobutil $\mathrm{CA}^{(17,25,26)}$.

\section{Utilidad en cierre de heridas en piel y mucosa}

El uso de los ACA para el cierre de heridas de origen traumático o quirúrgico ha sido ampliamente estudiado. Uno de los principios básicos del cierre de heridas en piel y mucosas es la adecuada aproximación de los bordes, minimizando la tensión. El método ideal del cierre debe ser fácil, seguro, rápido, de bajo costo, indoloro, sin inducir reacción local, alergia o infección, otorgando adecuados resultados estéticos ${ }^{(19,27)}$. El cierre de heridas con suturas permite un buen cierre, pero los tejidos pueden reaccionar localmente y estas requieren ser removidas posteriormente.

Para el cierre de heridas, los ACA generan un fuerte vínculo en sus bordes, lo que permite la adecuada cicatrización ${ }^{(11)}$. Tienen la ventaja de ser aplicados de forma más rápida que las suturas ${ }^{(28)}$, además de ser indoloros, sin necesidad de infiltración anestésica ni de ser removidos posteriormente de los tejidos, lo cual disminuye la incomodidad de los pacientes y el riesgo de heridas corto-punzantes ${ }^{(3,19,28)}$. Estas ventajas han demostrado ser de gran utilidad en pacientes pediátricos, reduciendo la ansiedad y miedo que generan el cierre de heridas y el posterior retiro de suturas ${ }^{(27)}$. Otra ventaja importante es que proporcionan una capa protectora resistente al agua, que sella las heridas de la exposición al agua y a la contaminación ${ }^{(11,19)}$.

Durante su polimerización, se produce una reacción exotérmica que generalmente no es percibida por el paciente ${ }^{(11)}$. Nuevas formulaciones como Glubran $2 \AA$, tendrían menos efectos térmicos locales, ya que polimeriza a $45^{\circ} \mathrm{C}$. Además, tiene mejores propiedades elásticas ${ }^{(29)}$.

La principal desventaja de este material es su reducida fuerza de tensión. Una revisión sistemática y meta análisis por Dumville et $a^{(28)}$ describió en 2014 que para el cierre de heridas en piel, los ACA presentaron mayor incidencia de dehiscencias al compararlos con las suturas. Por otro lado, no existieron diferencias en el rango de infección, apariencia estética, ni en el grado de satisfacción de cirujanos y pacientes. En general, los resultados estéticos obtenidos en el cierre de heridas cutáneas con ACA son equivalentes a los obtenidos con heridas suturadas $^{(19)}$, especialmente en pacientes más jóvenes ${ }^{(15)}$.

En el cierre de heridas cutáneas faciales con un mínimo de tensión, si bien se describen casos de dehiscencia y cicatriz hipertrófica, las diferencias no serían significativas, con buenos resultados estéticos ${ }^{(19)}$. Deorukhkar el at ${ }^{(30)}$, evaluaron en 2015 la utilidad de los ACA en el cierre de heridas faciales superficiales con baja tensión en niños. En ninguno de los casos se observó infección de la herida en el $1^{\circ}, 3^{\circ}$ y $7^{\circ}$ día del postoperatorio y sólo uno de los casos mostró dehiscencia de la herida en el $3^{\circ}$ día del postoperatorio. En general, no fue necesario inyectar anestésico local y la evaluación estética fue satisfactoria ${ }^{(30)}$.

En la mucosa oral, la calidad de los tejidos, la saliva, la vascularización y las funciones relacionadas con el habla, la masticación y la deglución ${ }^{(27)}$, influyen en que las suturas se puedan comportar de manera diferente. Los ACA parecen ser un método fácil, seguro, rápido e indoloro para el cierre de heridas intraorales no infectadas, creando una barrera contra la saliva(16).

En procedimientos quirúrgicos intraorales, los ACA presentan una mínima incidencia de dehiscencia y el resto de las complicaciones son similares a las habitualmente esperadas ${ }^{(3)}$. Si bien se ha descrito que causan menor malestar intraoperatorio y postoperatorio en los pacientes ${ }^{(16)}$, no se han observado diferencias significativas en la intensidad del dolor postoperatorio en el cierre de colgajos de exodoncias de terceros molares ${ }^{(14)}$.

\section{Efecto local en mucosa oral}

Los ACA son un material con índice de irritación oral mínimo ${ }^{(31)}$ Se ha descrito clínicamente mayor dolor y edema local asociados a las suturas en los tres primeros días postoperatorios ${ }^{(21)}$ y mayor inflamación e infiltrado de células inflamatorias cuando son comparadas con los ACA en heridas intraorales ${ }^{(16,32)}$. Por otro lado, los ACA estarían asociados a una mayor aparición de fibroblastos jóvenes, un menor tiempo de cicatrización y generarían una mejor epitelización( ${ }^{8,21)}$. (Figura 1)

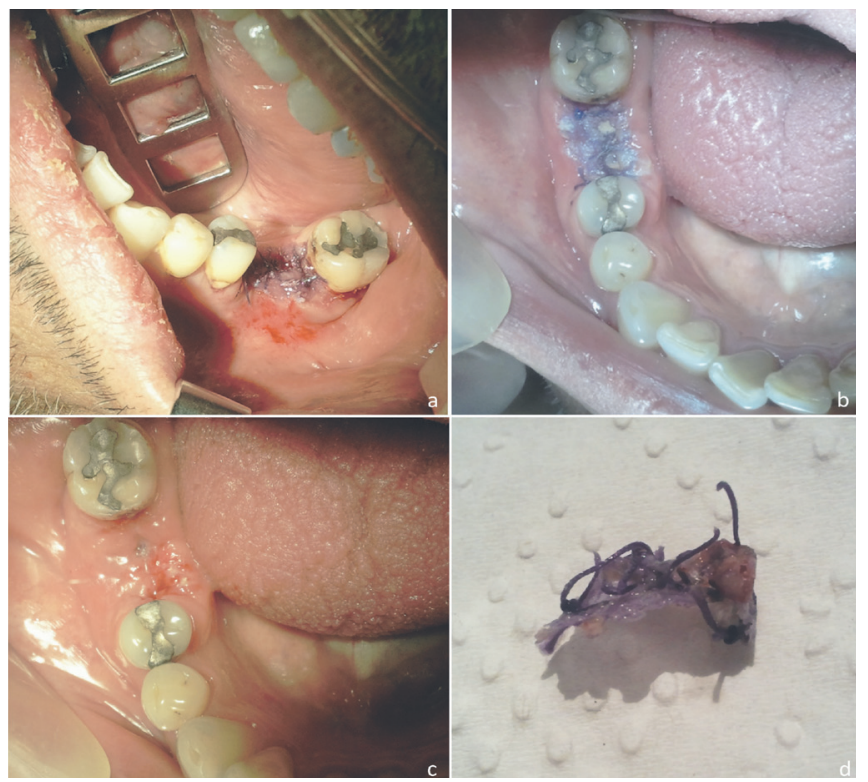

Figura 1. (a) Aplicación de adhesivo de cianiacrilato (Histocyl $\AA$ ) en el intraoperatorio, sobre sutura de exodoncia compleja. (b) Control postoperatorio a los 10 días, (c) herida operatoria luego de retiro de sutura y material adhesivo, (d) ACA retirado de la herida operatoria.

En un estudio clínico del año 1997, Giray et al comparó el cierre de heridas de mucosas orales con ACA y suturas de seda. No se observaron diferencias significativas en relación a hemorragia, epitelización, necrosis y formación de cicatrices. Sin embargo, el dolor $(p=0,023<0,05)$ y edema $(p=0,024<0,05)$ fueron significativamente mayores en los sitios suturados. La evaluación con microscopía electrónica al séptimo día del postoperatorio reveló una morfología normal de la ultra estructural en ambos lados ${ }^{(21)}$ Más recientemente, otro estudio clínico realizado en 2013, mostró que en pacientes sometidos a una apicetomía bilateral, las zonas cerradas con suturas desarrollaron inflamación de mayor intensidad, comparado con los lados cerrados con $\mathrm{ACA}^{(17)}$

El aumento de la respuesta inflamatoria en el lado suturado se podría atribuir a un aumento de la acumulación de placa, el trauma local y la reacción del tejido al material de sutura(16,17,21), mientras que los ACA actuarían como una costra, previniendo la infección secundaria y manteniendo una superficie donde la migración epitelial se produciría más fácilmente ${ }^{(32)}$

Por otro lado, algunos estudios han asociado el uso de ACA con mayores grados de infiltrado de polimorfonucleares e inflamación crónica a nivel subcutáneo hasta 45 días después de su aplicación ${ }^{\left({ }^{(3)}\right.}$ y una mayor respuesta tisular, con reacción granulomatosa a cuerpo extraño más pronunciada y retraso de la cicatrización $n^{(9,26,27,31,34)}$, en comparación con las suturas.

Javelet et al, en $1985^{(34)}$, comparó en un modelo animal el uso de suturas de seda versus la aplicación de ACA en heridas de mucosa oral. En el lado suturado, se observó una inflamación compuesta predominantemente por polimorfonucleares (PMN), leucocitos e histiocitos. Junto a los ACA, además de estas células inflamatorias, se encontraron células gigantes de cuerpo extraño. A las tres semanas las incisiones cerradas con ACA estaban más inflamadas, asociado este hallazgo con una respuesta lenta del tejido a la metabolización de cianoacrilato ${ }^{(34)}$. Más recientemente, un estudio clínico realizado por Vastani et al(16), describió mayor inflamación en el lado suturado en comparación con el lado cerrado con ACA, a las 2 semanas del post operatorio de una alveoloplastía a boca dividida ${ }^{(16)}$.

\section{Efecto antimicrobiano}

Varios autores han señalado las propiedades antibacterianas de los $\mathrm{ACA}^{(21,35,36)}$. Un estudio in vitro con isobutil-CA, mostró un efecto 
bacteriostático contra bacterias Gram positivas y destrucción activa de colonias de estreptococo en crecimiento, sin efecto contra bacterias Gram negativas ${ }^{(37)}$. Otro estudio en 1997, evaluó en un modelo animal el cierre de heridas contaminadas con octilo-CA en comparación a suturas monofilamento, obteniendo menor grado de infección clínico, histológico y bacteriológico a los 5 días con los ACA ${ }^{(35)}$. También tendrían un efecto en la reducción de la adherencia de la Cándida Albicans a células epiteliales bucales humanas in vitro(38).

El mecanismo antimicrobiano exacto de los ACA no está claro, pero es probable que su efecto se produzca en la pared celular, lo que explicaría que su acción esté limitada a organismos Gram-positivos. Una de las hipótesis plantea que la fuerte electronegatividad sobre el polímero podría reaccionar con la carga positiva de la pared celular de organismos Gram-positivos. Los organismos Gram negativos serían relativamente poco afectados debido a su membrana de polisacáridos, que aísla la célula de la pared ${ }^{(35)}$. Además, los ACA ofrecerían una barrera para los microorganismos en el sitio de la incisión, aislando los márgenes de la herida de la saliva y placa bacteriana ${ }^{(17,28,39)}$.

\section{Efecto hemostático}

Los ACA son utilizados para la embolización de vasos sanguíneos y son una opción descrita para el manejo de hemorragias secundarias a exodoncia de terceros molares ${ }^{(40)}$. Por otro lado, sus propiedades físicas han demostrado que parecen ser agentes hemostáticos efectivos a nivel local ${ }^{(3,26)}$.

En cirugía oral, los ACA permitirían logra una adecuada hemostasia inicial ${ }^{(8,41)}$ y un sangrado post operatorio menos significativo en los primeros días post operatorios ${ }^{(14)}$, en comparación al cierre sólo con suturas.

En un estudio realizado por Al-Belasy et al en $2003^{(10)}$, se evaluaron 30 pacientes usuarios de warfarina, que requerían exodoncia de piezas dentales con elevación de colgajo mucoperióstico. Para el cierre, además de suturas, en el grupo de estudio utilizaron ACA, logrando obtener una hemostasia completa más rápida que en el grupo control, donde sólo se utilizaron suturas, sin casos de sangrado postoperatorio ${ }^{(10)}$.

El mecanismo mediante el cual los ACA favorecen la hemostasia no está claro. Algunos autores plantean que formaría una película que bloquearía mecánicamente el flujo de sangre, sirviendo como superficie para que se active la cascada de la coagulación ${ }^{(10)}$. Estudios in vitro, han encontrado que los ACA inducen una disminución significativa del tiempo de tromboplastina parcial activada $(\text { TTPA })^{(42)}$; pero en la literatura no existen otros estudios más recientes que evalúen este efecto. (Figura 2).

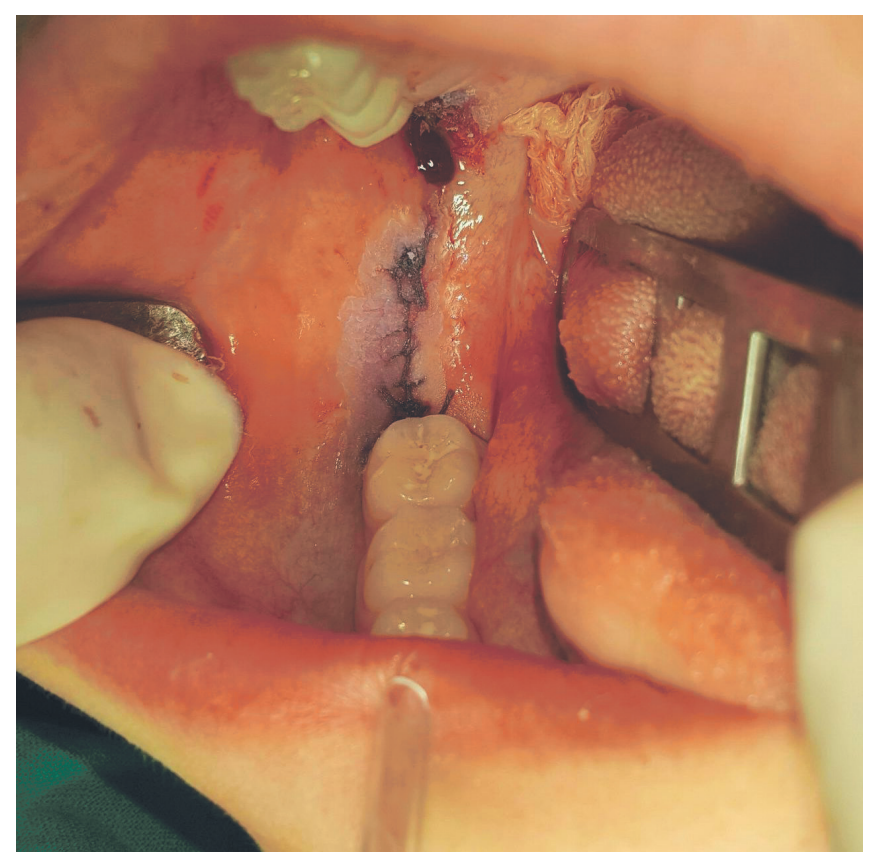

Figura 2. Aplicación de adhesivo de cianiacrilato (Histocyl $囚$ ), sobre herida suturada previamente, luego de exodoncia de terceros molares en mujer de 18 años con antecedentes de enfermedad de Von Willebrand.

\section{Otras aplicaciones en cirugía oral y maxilofacial}

Los ACA son una buena alternativa para el manejo de lesiones traumatológicas dentales, cuando no están disponibles los materiales habitualmente utilizados para su tratamiento. Si bien no es el material de elección para ferulizaciones, han demostrado ser útiles para inmovilizaciones dentales a corto plazo ${ }^{(43)}$.

También han demostrados resultados favorables en la fijación de insertos óseos en los rebordes alveolares. En modelos animales, se ha descrito que los injertos óseos fijados con Butil-CA, generan menor reabsorción en comparación a los injertos fijados con implantes de titanio y una mejor conservación del volumen del injerto en comparación al uso de fijación con tornillo(44). Los autores plantean que el trauma generado por la inserción de implantes o placas, podría inducir una reabsorción ósea inflamatoria que explicaría la diferencia en la preservación del volumen ${ }^{(45)}$.

Son una opción con excelentes resultados en los colgajos de reparación de fisuras labio-palatinas, sin evidencia de complicaciones como dehiscencia, hemorragia o infección a largo plazo. Además, al ser resistente al agua, no permiten el paso de partículas entre los lados orales y nasales del cierre quirúrgico ${ }^{(13)}$

Otras aplicaciones en el área de la cirugía oral y maxilofacial son la fijación de injertos gingivales, con una disminución del dolor en la zona receptora y una menor contracción del injerto ${ }^{(46)}$, el cierre de perforaciones de la membrana sinusal durante elevación de seno maxilar ${ }^{(47)}$, inmovilización de los cabos afrontados en la reparación de nervios periféricos ${ }^{(48)}$, y en el cierre de fistulas salivales parotídeas de difícil manejo ${ }^{(49)}$. El año 2013 Habib et al(12) publicó una serie de 165 casos, donde realizaron varios procedimientos maxilofaciales utilizando ACA, como fijación de injertos en piso de órbita y reborde alveolar, cierre de fisuras palatinas, reparación de fístulas orales, férulas nasales, manejo de dientes traumatizados y cierre de heridas, entre otros, sin evidencia de efectos adversos o respuesta inflamatoria importante ${ }^{(12)}$

\section{DISCUSIÓN}

El adhesivo ideal de los tejidos debe tener una mínima respuesta inflamatoria, ser efectivo, estable, biodegradable, no carcinogénico y debe liberar un mínimo de calor durante el proceso de polimerización después de su aplicación ${ }^{(27)}$. Aunque los resultados relacionados con su respuesta inflamatoria son controvertidos, frente a sus beneficios parece que sus efectos locales no san lo suficientemente importantes como para contraindicar su uso.

No existen estudios que evalúen el costo-beneficio del uso de ACA frente a materiales de suturas convencionales. El costo inicial es tres o más veces mayor para el uso de ACA, pero en algunas situaciones podrían contribuir a un ahorro global, ya que el uso de estos materiales toma menos tiempo ${ }^{(25)}$ y no requiere el uso de instrumental ni otros insumos, como los necesarios para el retiro de la sutura ${ }^{(19)}$. Si se tiene en cuenta el costo global del procedimiento quirúrgico, esta diferencia puede ser de menor importancia(28).

Es importante considerar que para una adecuada aplicación de estos materiales, es necesario que sean utilizados gota-a-gota, protegiendo los tejidos adyacentes, disponiendo de abundante suero fisiológico en caso de exposición accidental y evitando posible ingestión mediante una aspiración continua ${ }^{(12,29)}$. Las presentaciones de alta viscosidad pueden reducir el riesgo de migración del adhesivo fuera de la herida, (7,50). Independiente de esto, luego de su aplicación, el tejido debe ser inmovilizado por 30 segundos aproximadamente, para permitir que se complete la reacción de polimerización y deben eliminarse los excesos que puedan haber escurrido.

En base a lo descrito en la literatura, los ACA se plantean como una buena alternativa para el cierre de heridas de la mucosa oral. Aunque sus ventajas globales aún no superan al uso habitual de suturas, pueden ser una gran herramienta en ciertas situaciones clínicas, como pacientes pediátricos o pacientes que no están dispuestos a tolerar la anestesia local. Su potencial como agente hemostático y antibacteriano local parece de gran interés, pero es necesario que se realicen más estudios clínicos para consolidar sus aplicaciones y utilidades, por ejemplo, en pacientes con alteraciones de la coagulación o con mayor riesgo de infección de la herida operatoria.

\section{RESPONSABILIDADES ÉTICAS}

Protección de personas y animales. Los autores declaran que para esta investigación no se han realizado experimentos en seres humanos ni en animales.

Confidencialidad de los datos. Los autores declaran que han seguido los protocolos de su centro de trabajo sobre la publicación de datos de pacientes.

Derecho a la privacidad y consentimiento informado. Los autores han obtenido el consentimiento informado de los pacientes y/o sujetos referidos en el artículo. Este documento obra en poder del autor de correspondencia. 


\section{Bibliografía}

1. Ardis AE. US patent no 2467926 and 2467927 (1949).

2. Coover HW, Joyner FB, Shearer NG, et al. Chemistry and performance of cyanoacrylate adhesives. Soc Plast Eng J 15: 413, 1959.

3. Sagar P, Prasad K, Lalitha RM, Ranganath K. Cyanoacrylate for intraoral wound closure: a possibility? Int J Biomater. 2015;2015:1-6.

4. Pérez MDLC, Mayelin Guerra R, Fernández M, Márquez D, Velazco G, Rodríguez $\mathrm{J}$, et al. Effectiveness and safety of tisuacryl in treating dentin hypersensitivity (DH). MEDICC Rev. 2010;12(1):24-8.

5. Ylikontiola L, Sorsa T, Häyrinen-Immonen R, Salo T. Doxymycine-cyanoacrylate treatment of recurrent aphthous ulcers. Oral Surg Oral Med Oral Pathol Oral Radiol Endod. 1997 Mar;83(3):329-33

6. Wasson M, Ghodke B, Dillon JK. Exsanguinating hemorrhage following third molar extraction: Report of a case and discussion of materials and methods in selective embolization. J Oral Maxillofac Surg. 2012;70(10):227-5.

7. Blondeel PN, Murphy JW, Debrosse D, Nix JC, Puls LE, Theodore N, et al. Closure of long surgical incisions with a new formulation of 2-octylcyanoacrylate tissue adhesive versus commercially available methods. Am J Surg. 2004;188(3):307-13.

8. Camacho-Alonso F, López-Jornet P, Bermejo-Fenoll A. Effects of scalpel (with and without tissue adhesive) and cryosurgery on wound healing in rat tongues. Oral Surg Oral Med Oral Pathol Oral Radiol Endod. 2005;100(3):58-63.

9. Toriumi DM, Raslan WF, Friedman M, Tardy ME. Histotoxicity of cyanoacrylate tissue adhesives. A comparative study. Arch Otolaryngol Head Neck Surg. 1990;116(5):546-50.

10. Al-Belasy FA, Amer MZ. Hemostatic effect of n-butyl-2-cyanoacrylate (histoacryl) glue in warfarin-treated patients undergoing oral surgery. J Oral Maxillofac Surg. 2003;61(12):1405-9.

11. Buckley MJ, Beckman EJ. Adhesive use in oral and maxillofacial surgery. Oral Maxillofac Surg Clin North Am. 2010;22(1):195-9.

12. Habib A, Mehanna A, Medra A. Cyanoacrylate: a handy tissue glue in maxillofacial surgery: our experience in Alexandria, Egypt. J Maxillofac Oral Surg. 2013:12(3):243-7.

13. Turkaslan T, Ozcan H, Dayicioglu D, Ozsoy Z. Use of adhesives in cleft palate surgery: a new flap fixation technique. J Craniofac Surg. 2005 Jul;16(4):719-22. 14. Ghoreishian M, Gheisari R, Fayazi M. Tissue adhesive and suturing for closure of the surgical wound after removal of impacted mandibular third molars: A comparative study. Oral Surg Oral Med Oral Pathol Oral Radiol Endod.. 2009;108(1):e14-6. 15. Handschel JGK, Depprich RA, Dirksen D, Runte C, Zimmermann A, Kübler NR. A prospective comparison of octyl-2-cyanoacrylate and suture in standardized facial wounds. Int J Oral Maxillofac Surg. 2006;35(4):318-23.

16. Vastani A, Maria A. Healing of intraoral wounds closed using silk sutures and isoamyl 2-cyanoacrylate glue: A comparative clinical and histologic study. J Oral Maxillofac Surg. 2013;71(2):241-8.

17. Kumar MS, Natta S, Shankar G, Reddy SHK, Visalakshi D, Seshiah G V. Comparison between silk sutures and cyanoacrylate adhesive in human mucosa- a clinical and histological study. J Int oral Heal JIOH. 2013;5(5):95-100.

18. Inal S, Yilmaz N, Nisbet C, Güvenç T. Biochemical and histopathological findings of N-butyl-2-cyanoacrylate in oral surgery: an experimental study. Oral Surg Oral Med Oral Pathol Oral Radiol Endod. 2006 Dec;102(6):e14-7.

19. Soni A, Narula R, Kumar A, Parmar M, Sahore M, Chandel M. Comparing cyanoacrylate tissue adhesive and conventional subcuticular skin sutures for maxillofacial incisions - A prospective randomized trial considering closure time wound morbidity, and cosmetic outcome. J Oral Maxillofac Surg. 2013;71(12):2152. e1-2152.e8.

20. Leahey AB, Gottsch JD, Stark WJ. Clinical experience with N-butyl cyanoacrylate (Nexacryl) tissue adhesive. Ophthalmology. 1993 Feb;100(2):173-80.

21. Giray CB, Atasever A, Durgun B, Araz K. Clinical and electron microscope comparison of silk sutures and n-butyl-2-cyanoacrylate in human mucosa. Aust Dent J. 1997 Aug;42(4):255-8.

22. Cary R. Methyl cyanoacrylate and ethyl cyanoacrylate. Concise international chemical assessment document 36 . Inter-organizational program for the sound management of chemicals, UNEP/ ILO/WHO. Geneva: World Health Organization; 2001

23. Kaplan M, Oral B, Rollas S, Kut MS, Demirtas MM. Absorption of ethyl 2-cyanoacrylate tissue adhesive. Eur J Drug Metab Pharmacokinet. 2004 AprJun;29(2):77-81.

24. López-Jornet $P$, Camacho-Alonso F, Gómez-García F. Evaluation of biochemical the two cyanoacrylates: an experimental study in rats. J Biomater Appl. 2009 Sep;24(3):197-207.

25. Nagpal BM, Kumar G, Nagi GS, Singh P. Sutureless closure of operative skin wounds. Med J Armed Forces India. 2004;60(2):131-3.

26. Greer RO. Studies concerning the hsitotoxicity of isobutyl-2-cyanoacrylate tissue adhesive when employed as an oral hemostat. Oral Surg Oral Med Oral Pathol. 1975 Nov;40(5):659-69.
27. Muglali M, YImaz N, Inal S, Guvenc T. Immunohistochemical comparison of indermil with traditional suture materials in dental surgery. J Craniofac Surg. 2011;22(5):1875-9

28. Dumville J, Coulthard P, Worthington H V, Riley P, Patel N, Darcey J, et al. Tissue adhesives for closure of surgical incisions. Cochrane Database Syst Rev. 2014;(5):CD004287.

29. Burić $\mathrm{N}$. Use of $\mathrm{N}$-butyl cyanoacrylate with metacryloxisulfolane (glubran 2) surgical glue for flapless closure of oroantral communication. Implant Dent. 2013;22(3):238-43

30. Devrukhkar VN, Hegde RJ, Khare SS, Saraf TA. Evaluation of isoamyl 2-cyanoacrylate tissue adhesive in management of pediatric lacerations: An alternative to suturing. Ann Maxillofac Surg. 2015;5(1):49-54.

31. Garcia-simons G, Elena M, Graupera C, Hern A. Efecto del Tisuacryl sobre la mucosa oral. Rev CENIC. 2006:37(3):153-5.

32. Barreno AC, Vergiú CC, Malatto JR. Biocompatibilidad del cianoacrilato de butilo en suturas en piel en comparación con las suturas convencionales. Rev Odontológica Mex. 2013;17(2):81-90.

33. Moretti Neto RT, Mello I, Moretti ABDS, Robazza CRC, Pereira AAC. In vivo qualitative analysis of the biocompatibility of different cyanoacrylate-based adhesives. Braz Oral Res. 2008;22(1):43-7.

34. Javelet J, Torabinejad M, Danforth R. Isobutyl cyanoacrylate: A clinical and histologic comparison with sutures in closing mucosal incisions in monkeys. Oral Surgery, Oral Med Oral Pathol. 1985;59(1):91-4.

35. Quinn J, Maw J, Ramotar K, Wenckebach G, Wells G. Octylcyanoacrylate tissue adhesive versus suture wound repair in a contaminated wound model. Surgery. 1997;122(1):69-72.

36. Eiferman RA, Snyder JW. Antibacterial effect of cyanoacrylate glue. Arch Ophthalmol 1983:101(6):958-60. 10

37. Jandinski J, Sonis S. In vitro effects of isobutyl cyanocrylate on four types of bacteria. J Dent Res. 1971;50(6):1557-8.

38. McCarron PA, Donnelly RF, Canning PE, McGovern JG, Jones DS. Bioadhesive, non-drug-loaded nanoparticles as modulators of candidal adherence to buccal epithelial cells: A potentially novel prophylaxis for candidosis. Biomaterials. 2004;25(12):2399-407

39. Dip EC, Luz D, Castro-Silva I, Pires A, Linhares A, Granjeiro M. Hypernociception and wound healing after application of cyanoacrylate ester as a tissue adhesive in rats. Oral Surg Oral Med Oral Pathol Oral Radiol. 2012 Nov;114(5 Suppl):S79-85.

40. Sagara Y, Kiyosue H, Tanoue S, Shimada R, Hongo N, Kohno T, et al. Selective transarterial embolization with n-butyl-2-cyanoacrylate for the treatment of arterial hemorrhage after third molar extraction. Neuroradiology. 2013;55(6):725-31.

41. Pérez M, Fernández I, Márquez D, Bretaña RM. Use of N-butyl-2-cyanoacrylate in oral surgery: biological and clinical evaluation. Artif Organs. 2000 Mar;24(3):241-3. 42. Montanaro L, Arciola CR, Cenni E, Ciapetti G, Savioli F, Filippini F, et al. Cytotoxicity, blood compatibility and antimicrobial activity of two cyanoacrylate glues for surgical use. Biomaterials. 2001;22(1):59-66.

43. Cobb ARM, Ahmad S, Kumar M. Use of n-butyl 2-cyanoacrylate tissue adhesive to splint traumatised teeth in the emergency department. $\mathrm{Br} \mathrm{J}$ Oral Maxillofac Surg. 2011:49(6):483-5.

44. Salata LA, Mariguela VC, Antunes AA, Grossi-Oliveira G, Almeida A, Taba M. Short-term evaluation of grafts fixed with either $\mathrm{N}$-butyl-2-cyanocrylate or screws. $\mathrm{J}$ Oral Maxillofac Surg. 2014;72(4):676-82.

45. de Oliveira Neto PJ, Cricchio G, Hawthorne AC, Okamoto R, Sennerby L, Lungren S, et al. Tomographic, histological, and immunohistochemical evidences on the use of N-butyl-2-cyanoacrilate for onlay graft fixation in rabbits. Clin Implant Dent Relat Res. 2012 Dec;14(6):861-71

46. Gümüs P, Buduneli E. Graft stabilization with cyanoacrylate decreases shrinkage of free gingival grafts. Aust Dent J. 2014;59(1):57-64

47. Choi BH, Kim BY, Huh JY, Lee SH, Zhu SJ, Jung JH, et al. Cyanoacrylate adhesive for closing sinus membrane perforations during sinus lifts. J CranioMaxillofacial Surg. 2006;34(8):505-9

48. Landegren T, Risling M, Persson JKE, Sondén A. Cyanoacrylate in nerve repair transient cytotoxic effect. Int J Oral Maxillofac Surg. 2010 Jul;39(7):705-12.

49. Pesic Z, Buric N, Vuckovic I, Petrovic D, Krasic D, Cosic A, et al. Use of 2-OctylCyanoacrylate in surgical closing of Postparotidectomy salivary fistulas. Eur Arch Otorhinolaryngol. 2011;268(11):1691-4.

50. Sniezek PJ, Walling HW, DeBloom JR, Messingham MJ, VanBeek MJ, Kreiter $\mathrm{CD}$, et al. A randomized controlled trial of high-viscosity 2-octyl cyanoacrylate tissue adhesive versus sutures in repairing facial wounds following Mohs micrographic surgery. Dermatol Surg. 2007 Aug;33(8):966-71. 